\title{
Performance Analysis of an Optimal Circular 16-QAM for Wavelet Based OFDM Systems
}

\author{
Khaizuran ABDULLAH, Seedahmed S. MAHMOUD*, Zahir M. HUSSAIN \\ School of Electrical \& Computer Engineering, RMIT University, Melbourne, Australia \\ *Future Fiber Technologies Pty. Ltd., Mulgrave, Australia \\ E-mail:khaizuran.abdullah@rmit.edu.au,smahmoud@fft.com.au,zmhussain@ieee.org \\ Received August 20, 2009; revised September 29, 2009; accepted October 29, 2009
}

\begin{abstract}
The BER performance for an optimal circular 16-QAM constellation is theoretically derived and applied in wavelet based OFDM system in additive white Gaussian noise channel. Signal point constellations have been discussed in much literature. An optimal circular 16-QAM is developed. The calculation of the BER is based on the four types of the decision boundaries. Each decision boundary is determined based on the space distance $\mathrm{d}$ following the pdf Gaussian distribution with respect to the in-phase and quadrature components $\mathrm{nI}$ and nQ with the assumption that they are statistically independent to each other. The BER analysis for other circular M-ary QAM is also analyzed. The system is then applied to wavelet based OFDM. The wavelet transform is considered because it offers a better spectral containment feature compared to conventional OFDM using Fourier transform. The circular schemes are slightly better than the square schemes in most SNR values. All simulation results have met the theoretical calculations. When applying to wavelet based OFDM, the circular modulation scheme has also performed slightly less errors as compared to the square modulation scheme.
\end{abstract}

Keywords: Performance OFDM, Fourier-Based OFDM, Wavelet-Based OFDM, Circular 16-QAM, Square 16-QAM

\section{Introduction}

Quadrature amplitude modulation (QAM) is one of the most popular modulation schemes used by orthogonal frequency division multiplexing. Some popular types of M-ary QAM are 4-QAM, 16-QAM and 64-QAM. The number of 4,16 and 64 is corresponding to $2^{2}, 2^{4}$ and $2^{6}$ in which that the superscript number 2, 4 or 6 is the bit rate per OFDM symbol respectively. In this paper, the constellation points derivation and the BER analysis are focused on 16-QAM, which gives an intermediate result of BER performance between 4- and 64-QAM in an AWGN channel [1]. The 16-QAM is also one of the standard modulation schemes in OFDMs' applications such as terrestrial Digital Video Broadcasting (DVB), Digital Audio Broadcasting (DAB) and High Performance Radio LAN Version 2 (HIPERLAN/2) [2]. In the transmitter, an OFDM symbol is mapped from binary to complex signal with amplitude and phase represented in real and imaginary number. On the other hand, the signal is demapped or extracted from complex signal to OFDM symbol in the receiver. The decision boundary is needed to detect the correct symbols between the transmitter and receiver. The bit error rate (BER) performance is determined after performing the difference of errors between the transmitted bits with the received bits. The BER performance of M-ary QAM has been investigated by several authors. The exact BER expressions for QAM is presented in [3]. An extension of BER expressions considering of an arbitrary constellation size is discussed in [4]. Both works include the square constellation points.

In this paper, we propose an alternative BER expression using optimal circular constellation points. The calculation of probability of error is based on determining the decision boundary. We have proposed four types of decision boundaries.

Based on these types, the probability of error occurs when the receiver is making an incorrect decision. To the best of our knowledge, there is no work of the probability of error calculation for a circular 16-QAM with the application of wavelet-based OFDM, namely discrete wavelet transform (DWT). The principle feature of DWT is it has low pass and high pass filters satisfying perfect reconstruction property in the transmitter and receiver 
[5-7]. The use of wavelet is significant since wavelet has a better spectral containment feature compared to conventional OFDM using Fourier filter [8,9]. To be specific, the application of Mband wavelet filters in wavelet-based OFDM, having the pulses for different overlapping data blocks in time, is designed to achieve a combination of subchannel spectral containment and bandwidth efficiency that is fundamentally better than with other forms of multicarrier modulation [9]. Other term of DWT is discrete wavelet multitone modulation (DWMT) or wavelet-OFDM (W-OFDM).

This paper is organized as follows. Determining the constellation points the circular 16-QAM is discussed in the next section followed by the calculation of an exact probability of error in Section 3 and the wavelet OFDM principles in Section 4. The system model of wavelet based OFDM is discussed in Section 5 and finally the BER results are obtained in Section 6.

\section{The Derivation of an Optimal Circular Constellation Points}

A circular signal point constellation has been discussed in [1]. However, the discussion is for $M=8$ constellations, while $M=16$ can be inferred as sub-optimal. We extend the work for an optimal circular 16-QAM. In this section, we discuss only the derivation for the circular 16-QAM since the derivation for a square 16-QAM is well known in many literatures. The number of circles and amplitudes for the circular scheme is different than those of the conventional square scheme. Let the number of circles define as $S$ and the amplitude level associating with the diameter define as $r$. In this particular circular 16-QAM, we have $S=4$ with 4 points on all circles with different diameter $r_{1}, r_{2}, r_{3}$ and $r_{4}$ with the derivation as follows

$$
\begin{aligned}
& r_{1}=\sqrt{d^{2}+d^{2}}=\sqrt{2} \\
& r_{2}=\sqrt{3 d}=\sqrt{3} \\
& r_{3}=\sqrt{\left(1+r_{2}\right)^{2}+2^{2}-4\left(1+r_{2}\right) \cos \left(P_{h}\right)} \\
& r_{4}=\sqrt{d_{s}^{2}+r_{1}^{2}-2 d_{s} r_{1} \cos \left(\frac{P_{p}}{2}+P_{s i}\right)}
\end{aligned}
$$

where $P_{h}=\frac{\pi}{3}+P_{0}, P_{0}=\tan ^{-1}\left(\frac{1}{r_{2}}\right), d_{s}=\sqrt{8 d^{2}-(8 d) \cos (b)}$, $b=\pi-P_{p}, P_{p}=\phi-\frac{\pi}{3}, \phi=2 \pi-2 P_{s i}$ and $P_{s i}=\pi-\frac{\pi}{4}-P_{0}$.

Note that the minimum distance is $d=1$. By rearranging (1) in vector representation, we have

$$
V_{c}=d \times\left[\begin{array}{llll}
r_{1} & 1+r_{2} & r_{3} & r_{4}
\end{array}\right]
$$

Since every 4 points share one diameter, we repeat every amplitude 4 times. Therefore

$$
v_{c}=\left(V_{c}\right)^{T} \times\left[\begin{array}{llll}
1 & 1 & 1 & 1
\end{array}\right]
$$

and the amplitude vector $A_{v c}$ for all amplitudes of QAM constellation points becomes

$$
A_{v c}=\left(v_{c}\right)^{T}
$$

Subsequently we need to derive the rotating phase for the constellation points. Thus,

$$
\begin{aligned}
\theta_{\mathrm{c} 1} & =\frac{\pi}{4} \times\left[\begin{array}{llllllll}
1 & 3 & 5 & 7 & 0 & 2 & 4 & 6
\end{array}\right] \\
\theta_{\mathrm{c} 2} & =\left[\begin{array}{llll}
h_{0} & h_{1} & h_{2} & h_{3}
\end{array}\right] \\
\theta_{c 3} & =\left[\begin{array}{llll}
g_{0} & g_{1} & g_{2} & g_{3}
\end{array}\right]
\end{aligned}
$$

where

$h_{0}=\sin ^{-1}\left(\frac{2}{r_{3}} \sin \left(p_{h}\right)\right), h_{1}=\pi-h_{0}, h_{2}=\pi+h_{0}, h_{3}=-h_{0}$, $g_{0}=\frac{\pi}{4}+\sin ^{-1}\left(\frac{d_{s}}{r_{4}} \sin \left(\frac{P_{p}}{2}+P_{s i}\right)\right), g_{1}=\pi-g_{0}, g_{2}=\pi+g_{0}$, $g_{3}=-g_{0}$. Rearranging (5), in vector representation, we obtain

$$
\theta_{c}=\left[\begin{array}{lll}
\theta_{c 1} & \theta_{c 2} & \theta_{c 3}
\end{array}\right]
$$

and $\theta_{c}$ has all angles of all constellation points. Combining the amplitude $A_{v c}$ and the phase $\theta_{c}$, the circular 16-QAM (Scir) is expressed as

$$
S_{c i r}=A_{v c} \cos \left(\theta_{c}\right)+j A_{v c} \sin \left(\theta_{c}\right)
$$

The simulation result is obtained and shown in Figure 1.

\section{The Exact BER Calculation}

Each decision boundary in Figure 2 is determined by the space distance $d$ following the pdf Gaussian distribution with respect to the in-phase and quadrature components

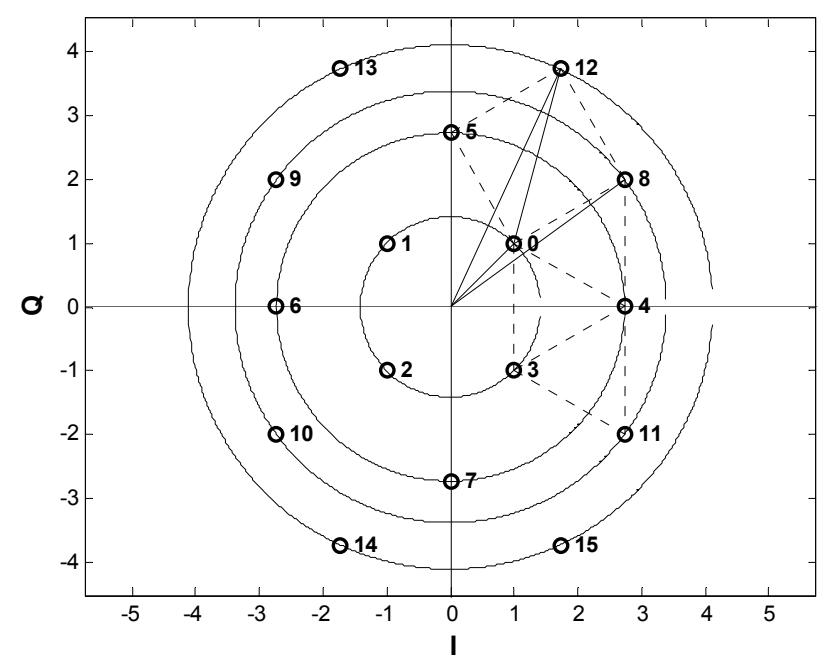

Figure 1. Circular 16-QAM constellation points. 
$n_{I}$ and $n_{Q}$ with the assumption that they are statistically independent to each other. Thus, four types of boundary regions can be determined accordingly from the figure. Type 1 in part (a) of Figure 3 is for the points related to the most inner circles in Figure 2. The probability of a correct decision is

$$
\begin{aligned}
P_{c 1}= & {\left[1-\left\{P\left(n_{I} \leq-\frac{0.5 d}{\sigma}\right)+P\left(n_{I} \geq \frac{0.5 d}{\sigma}\right)\right\}\right] } \\
& \times\left[1-\left\{P\left(n_{Q} \geq \frac{0.5 d}{\sigma}\right)+P\left(n_{I} \leq-\frac{0.5 d}{\sigma}\right)\right\}\right] \\
= & {\left[1-2 Q\left(\frac{0.5 d}{\sigma}\right)\right]\left[1-2 Q\left(\frac{0.5 d}{\sigma}\right)\right] }
\end{aligned}
$$

where $\mathrm{Q}(\mathrm{x})=\frac{1}{\sqrt{2 \pi}} \int_{\mathrm{x}}^{\infty} \mathrm{e}^{\frac{\mathrm{x}^{2}}{2}} \mathrm{dx}$ and the probability of error is

$$
P_{e 1}=4\left[1-P_{c 1}\right]=4\left[1-\left(1-2 Q\left(\frac{0.5 d}{\sigma}\right)\right)^{2}\right]
$$

The next type is associated with 2 points, $\{5,7\}$. The boundary region is shown in part (b) of Figure 3. The probability of correct decision can be expressed as

$$
\begin{aligned}
P_{c 2}= & {\left[1-\left\{P\left(n_{I} \leq-\frac{0.5 d}{\sigma}\right)+P\left(n_{I} \geq \frac{0.5 d}{\sigma}\right)\right\}\right] } \\
& \times\left[1-P\left(n_{Q} \leq-\frac{0.232 d}{\sigma}\right)\right] \\
= & {\left[1-2 Q\left(\frac{0.5 d}{\sigma}\right)\right]\left[1-Q\left(\frac{0.232 d}{\sigma}\right)\right] }
\end{aligned}
$$

and the probability of error for Type 2 is given by

$$
\begin{aligned}
P_{e 2} & =2\left[1-P_{c 1}\right] \\
& =2\left[1-\left\{\left[1-2 Q\left(\frac{0.5 d}{\sigma}\right)\right]\left[1-Q\left(\frac{0.232 d}{\sigma}\right)\right]\right\}\right]
\end{aligned}
$$

Considering the BER analysis for Type 3 , six points $\{4,6,8,9,10,11\}$ are involved. The decision boundary related to this type is shown in part (c) of Figure 3. Then probability of correct decision is given by

$$
\begin{aligned}
P_{c 3}= & {\left[1-\left\{P\left(n_{Q} \geq \frac{0.5 d}{\sigma}\right)+P\left(n_{Q} \leq-\frac{0.5 d}{\sigma}\right)\right\}\right] } \\
& \times\left[1-P\left(n_{I} \leq-\frac{1.232 d}{\sigma}\right)\right] \\
= & {\left[1-2 Q\left(\frac{0.5 d}{\sigma}\right)\right]\left[1-Q\left(\frac{1.232 d}{\sigma}\right)\right] }
\end{aligned}
$$

and the probability of error is

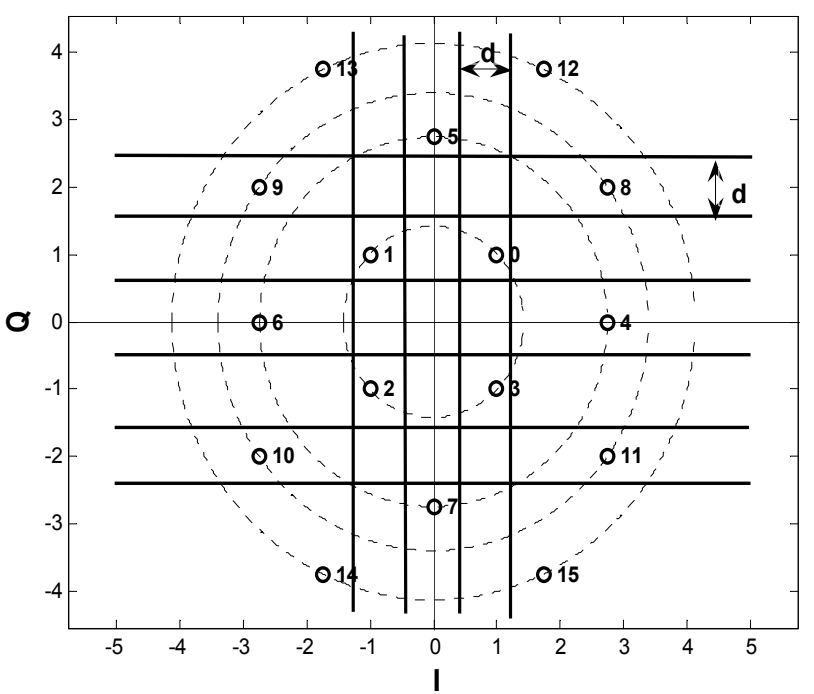

Figure 2. Signal-space diagram for circular 16-QAM.

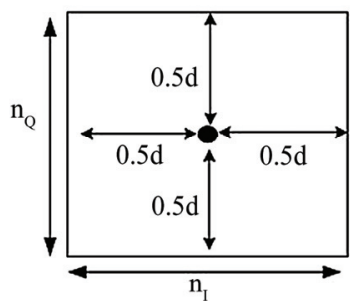

(a) Type 1

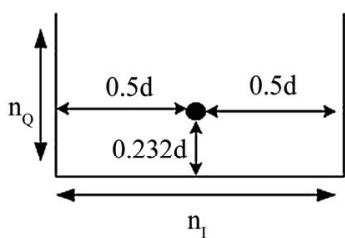

(b) Type 2

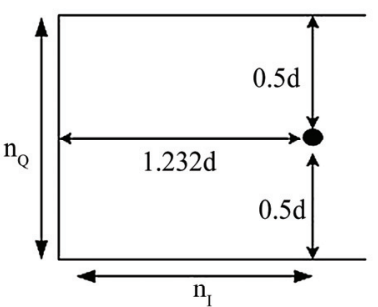

(c) Type 3

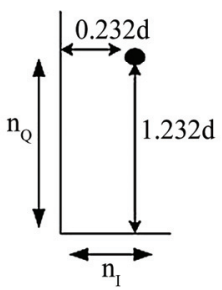

(d) Type 4
Figure 3. All types of decision boundary associated to Figure 2. Note that $0.5,0.232$ and 1.232 are the results obtained from (7) due to variations of $A_{v c}$ and $\theta_{c}$.

$$
\begin{aligned}
P_{e 3} & =6\left[1-P_{c 3}\right] \\
& =6\left[1-\left\{\left[1-2 Q\left(\frac{0.5 d}{\sigma}\right)\right]\left[1-Q\left(\frac{1.232 d}{\sigma}\right)\right]\right\}\right]
\end{aligned}
$$

Next, the decision boundary for Type 4 . It is associated to the points $\{12,13,14,15\}$. The probability of correct decision is

$$
\begin{aligned}
P_{c 4} & =\left[1-P\left(n_{I} \leq-\frac{0.232 d}{\sigma}\right)\right]\left[1-P\left(n_{Q} \leq-\frac{1.232 d}{\sigma}\right)\right] \\
& =\left[1-Q\left(\frac{0.232 d}{\sigma}\right)\right]\left[1-Q\left(\frac{1.232 d}{\sigma}\right)\right]
\end{aligned}
$$

and the probability of error for Type 4 is expressed as 


$$
\begin{aligned}
P_{e 4} & =4\left[1-P_{c 4}\right] \\
& =4\left[1-\left\{\left[1-Q\left(\frac{0.232 d}{\sigma}\right)\right]\left[1-Q\left(\frac{1.232 d}{\sigma}\right)\right]\right\}\right]
\end{aligned}
$$

Combining and rearranging Equations (9), (11), (13) and (15), the average probability of error for the circular 16-QAM scheme is given by

$$
\begin{aligned}
P_{c i r} & =\frac{1}{16}\left[P_{e 1}+P_{e 2}+P_{e 3}+P_{e 4}\right] \\
& =\frac{1}{8}\left[8 A\left(2-A-\frac{1}{4}(B+3 C)\right)+2 B\left(\frac{3}{2}-C\right)+5 C\right]
\end{aligned}
$$

where $\mathrm{A}=\mathrm{Q}\left(\frac{0.5 \mathrm{~d}}{\sigma}\right), \mathrm{B}=\mathrm{Q}\left(\frac{0.232 \mathrm{~d}}{\sigma}\right) \quad$ and $\mathrm{C}=\mathrm{Q}\left(\frac{1.232 \mathrm{~d}}{\sigma}\right)$. Using $\mathrm{d}=\sqrt{\frac{3 \log _{2} \mathrm{M} \cdot \mathrm{E}_{\mathrm{b}}}{2(\mathrm{M}-1)}}$ from [4] where $M=16$ and $\sigma=\frac{1}{2} \sqrt{\frac{\mathrm{N}_{0}}{5}}$, Equation (16) can be expressed as in term of energy per bit over noise density ratio $\left(\frac{\mathrm{E}_{\mathrm{b}}}{\mathrm{N}_{0}}\right)$.

Thus parameters $\mathrm{A}, \mathrm{B}$ and $\mathrm{C}$ can be rewritten as

$$
\mathrm{A}=\mathrm{Q}(0.5(\gamma)), \mathrm{B}=\mathrm{Q}(0.232(\gamma))
$$

and

$$
\mathrm{C}=\mathrm{Q}(1.232(\gamma)) \text { where } \gamma=4 \sqrt{\frac{1}{2} \frac{E_{b}}{N_{0}}} .
$$

The analysis can also determine the exact BER for other circular M-ary QAM. Note that the process of obtaining BER analysis for the square scheme is excluded since it is available in much literature.

\section{Wavelet OFDM Principles}

A wavelet is normally assigned the square integrable function $\psi(\mathrm{t})$ to illustrate the wavelet fundamental definition [10]. In other literature [7], it is also indicated by $\psi(\mathrm{t}) \in L^{2}(R)$ where $L$ is a Lebesque integral and 2 signifies the integral of the square of the modulus of the function, and $\mathrm{R}$ denotes the real number for integration of the independent variable $t$. In this section, we discuss two principles of wavelet transforms, orthogonal and biorthogonal wavelet as follows.

\subsection{Orthogonal Wavelets}

The Fourier transform has exponential parts consisting of cosine and sine signal bases. These bases are orthogonal to each other. The wavelet transform also has orthogonal bases. Its bases are low pass and high pass filters which are associated with the scaling and wavelet functions respectively. Among orthogonal wavelets are Daubechies, Coiflets, Morlet and Meyer [6].

Orthogonal wavelet functions can be generated by scaling and shifting properties as follows [10]:

$$
\psi_{a b}(t)=\frac{1}{\sqrt{2}} \psi\left(\frac{t-b}{a}\right)
$$

where $a$ and $b$ are the scaling and shifting real parameter values. According to [11], the wavelet transform is called continuous if $a$ and $b$ are continuous. The drawbacks of a continuous wavelet transform are redundancy and impracticality. To avoid these problems, those parameters have to be discredited as follows $[10,12]$ :

$$
\begin{aligned}
& a=a_{0}^{m} \\
& b=n b_{0} a
\end{aligned}
$$

where $m$ and $n$ indicates the exponential integers. From (17) and (18), the basis of the DWT can be formed as

$$
\psi_{m n}(t)=a_{0}^{-\frac{m}{2}} \psi\left(a_{0}^{-m} t-n b_{0}\right)
$$

Using $a_{0}=2$ and $b_{0}=1$, we can have the signal function

$$
\begin{aligned}
U(t) & =\sum_{n=-\infty}^{\infty} C_{L, n} \phi\left(2^{-L} t-n\right) \\
& +\sum_{m=1}^{L} \sum_{n=-\infty}^{\infty} D_{m n} 2^{-\frac{m}{2}} \psi\left(2^{-m} t-n\right)
\end{aligned}
$$

where the scaling coefficient $C_{L . n}$ is

$$
\begin{aligned}
C_{L . n} & =\left\langle U(t), \phi_{L, n}(t)\right\rangle \\
& =2^{-\frac{L}{2}} \int U(t) \phi\left(2^{-L} t-n\right) d t
\end{aligned}
$$

where $\phi_{\mathrm{L}, \mathrm{n}}(t)=2^{-\frac{L}{2}} \phi\left(2^{-L} t-n\right)$ and the wavelet coefficient $D_{m n}$ is

$$
\begin{aligned}
D_{m n} & =\left\langle U(t), \psi_{m n}(t)\right\rangle \\
& =2^{-\frac{L}{2}} \int U(t) \psi\left(2^{-L} t-n\right) d t
\end{aligned}
$$

In (20), the time domain signal $U(t)$ is DWT transformed to scales in which all the coefficients are denoted as the scales [10]. $U(t)$ can also be called the finite resolution wavelet representation [12]. The sum of scaled $\varphi(2 t)$ can make up the parent scaling function, and can be expressed as $[7,10]$ :

$$
\phi(t)=\sqrt{2} \sum_{n} h_{n} \phi(2 t-n)
$$


where the coefficients $h_{n}$ are a sequence of real or perhaps complex numbers called the scaling function (or scaling vector or filter). The use of $\sqrt{2}$ is to maintain the norm of the scaling function with the scale of 2. This scaling function in (23) can also be used for the multiresolution analysis (MMRA) [13]. A fundamental wavelet function can be expressed as a linear combination of translates of the scaling function as follows $[10,12]$ :

$$
\psi(t)=\sqrt{2} \sum_{n} g_{n} \phi(2 t-n)
$$

where the wavelet coefficients $g_{n}$ are related to the scaling coefficients $h_{n}$ by

$$
g(n)=(-1)^{n} h_{1-n}
$$

An example of the application of (23) is the Haar scaling function which is given by [7] as follows:

$$
\phi_{H}(t)=\phi(2 t)+\phi(2 t-1)
$$

It can be seen that $\phi(2 t)$ can be used to construct $\phi_{H}(t)$. It also can be noted that (26) is the result of (23) for the first 2 sequence of discrete samples of $n$ with coefficients $h(0)=\frac{1}{\sqrt{2}}, h(1)=\frac{1}{\sqrt{2}} \quad$ [7]. Examples of Haar scaling and wavelet functions are shown in Figure 4.

The Haar wavelet can be categorised as an orthogonal wavelet. All Daubechies wavelet families are categorised as orthogonal wavelets [6]. Another figure of a Daubechie wavelet such as db2 is shown in Figure 5.

\subsection{Biorthogonal Wavelets}

Biorthogonal wavelets are different than orthogonal wavelets because they have biorthogonal bases. Their bases have symmetric perfect reconstruction properties with compactly support. They also have two duality functions for each scaling and wavelet functions which are $\phi$ and $\hat{\phi}$ for the scaling filters, and $\psi$ and $\hat{\psi}$ for the wavelet filters accordingly. In MATLAB, we have built-in functions such as bior1.1, bior2.2, bior5.5, rbio1.1, rbio2.2 and rbio5.5. The number next to the wavelet name refers to the length of the filter in the decomposition and reconstruction filters respectively.

Biorthogonal wavelets can be constructed from orthogonal wavelets by considering the duality concept. Let $\phi$ and $\hat{\phi}$ be two scaling functions and let $\psi$ and $\hat{\psi}$ be two wavelet functions, then we can express the biorthogonal scaling and wavelet functions as follows $[5,14]$ :

$$
\begin{aligned}
& \langle\phi(t), \hat{\phi}(t)\rangle=\delta_{n} \\
& \langle\psi(t), \hat{\psi}(t)\rangle=\delta_{k} \\
& \langle\psi(t), \hat{\phi}(t)\rangle=0 \\
& \langle\hat{\psi}(t), \phi(t-n)\rangle=0
\end{aligned}
$$

where $\hat{\phi}(t)=\sqrt{2} \sum_{n} \hat{h}_{n} \hat{\phi}(2 t-n)$ and $\hat{\psi}(t)=\sqrt{2} \sum_{n} \hat{h}_{n} \hat{\psi}(2 t-n)$

with $\delta_{n}$ and $\delta_{k}$ are the results of biorthogonal bases. The last two equations in (27) satisfy the orthogonality properties. One advantage of using biorthogonal wavelets is that the scaling and wavelet functions are symmetric due to the duality concept $[5,7]$, therefore, biorthogonal wavelets provide an advantage over orthogonal
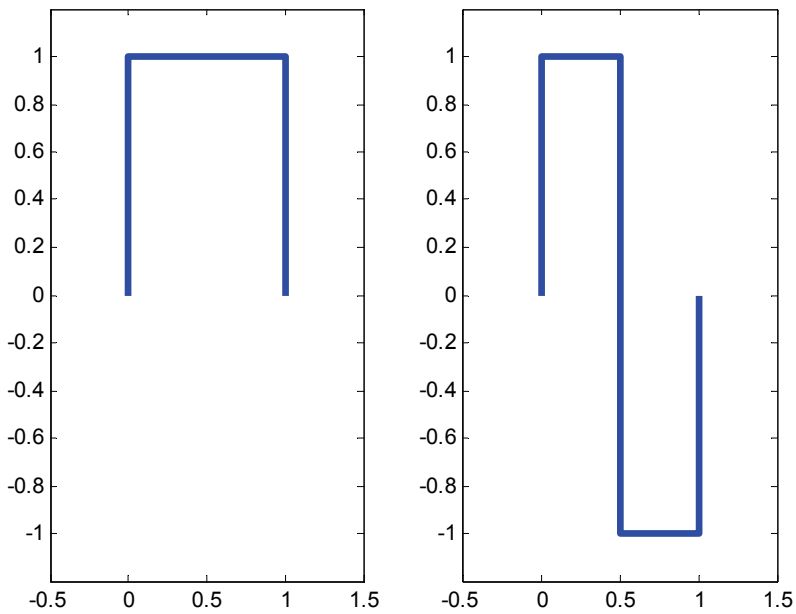

Figure 4. Haar (db1) scaling function $\phi(t)$ (left) and wavelet function $\psi(t)$ (right).
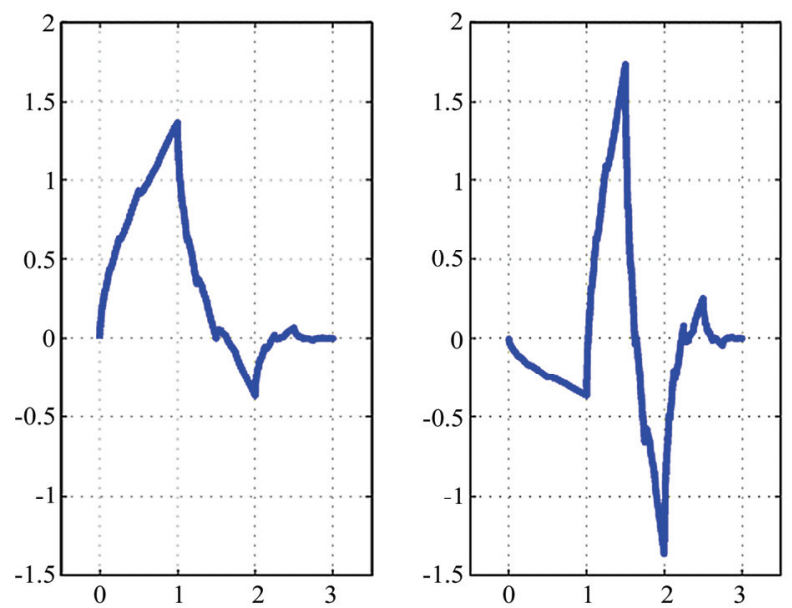

Figure 5. Db2 scaling function $\phi(t)$ (left) and wavelet function $\psi(t)$ (right). Note that this plot is similar to [5] p. 197 and [7] p. 81. 

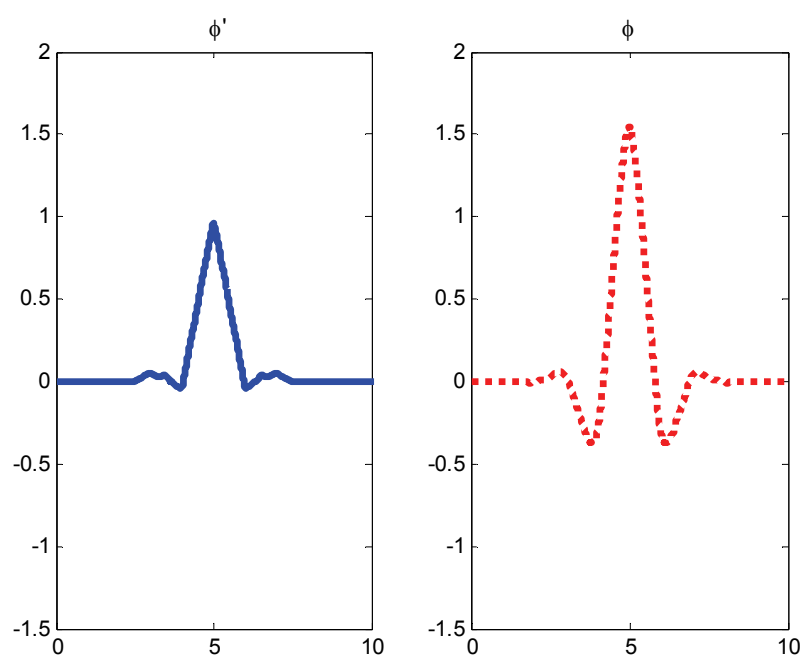

Figure 6. Bior5.5 shows duality concept with two scaling functions, $\hat{\phi}$ (left) and $\phi$ (right). Note that this plot is similar to [5] p. 280.
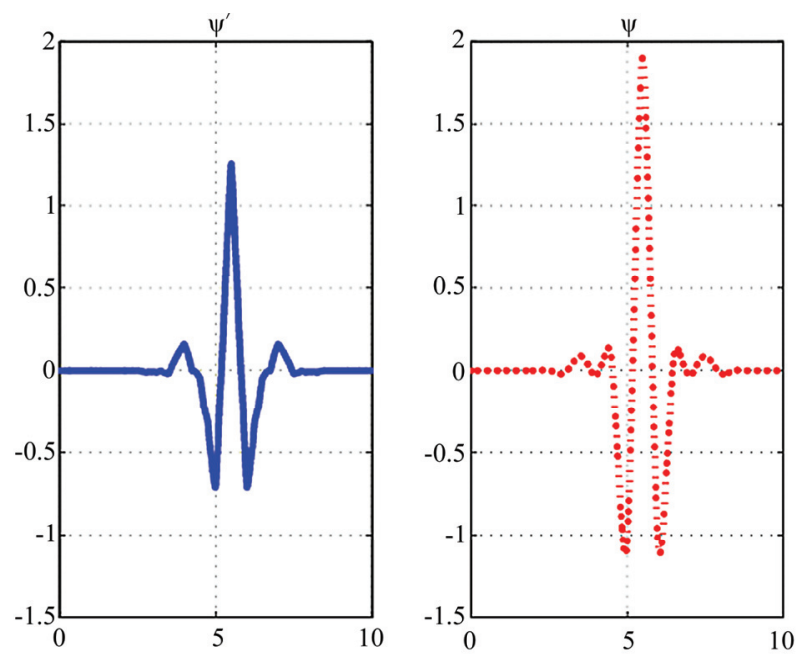

Figure 7. Bior5.5 shows duality concept with two wavelet functions, $\hat{\psi}(t)$ (left) and $\psi(t)$ (right). Note that this plot is similar to [5] p. 280.

wavelets because they offer not only orthogonality but also symmetry. In [15], comparing orthogonal transforms, biorthogonal transforms relax some of the constraints on the mother wavelet (or filters) and allow the mother wavelet to be symmetric and have linear phase. The plots of biorthogonal scaling and wavelet functions are shown in Figure 6 and Figure 7.

\section{System Model of Wavelet-Based OFDM}

The wavelet transform blocks comprise of an inverse discrete wavelet transform (IDWT) at the transmitter and a discrete wavelet transform (DWT) at the receiver as shown in Figure 8. Due to the overlapping nature of wavelets, the wavelet-based OFDM does not need a cyclic prefix to deal with the delay spreads of the channel. As a result, it has a higher spectral containment than in Fourier based OFDM [8,9]. The DWT-OFDM system model comprise of low pass as LPF filter coefficients and $h$ as HPF filter coefficients, the orthonormal bases are satisfied by four possible ways as follows [6]:

$$
\begin{aligned}
& \left\langle g, g^{*}\right\rangle=1 \\
& \left\langle h, h^{*}\right\rangle=1 \\
& \left\langle g, h^{*}\right\rangle=0 \\
& \left\langle h, g^{*}\right\rangle=1
\end{aligned}
$$

where (28) or (29) is related to the normal property and (30) or (31) is for orthogonal property accordingly. The commas and star symbols in Equations (28) to (31) above are referring to the dot product and transpose vector accordingly. Both filters are assumed having perfect reconstruction property. This means that the input and output of the two filters are expected to be the same. The $g$ and $h$ coefficients can be further described as having convolution operations to perform as orthonormal wavelets which can be represented as [16]

$$
\begin{aligned}
\alpha_{i}(n)= & h\left(\frac{n}{2^{i}}\right) * g\left(\frac{n}{2^{i}}\right) * \ldots * g\left(\frac{n}{2^{i-j}}\right) * \ldots * g(n) \\
\alpha_{N-1}(n) & =g\left(\frac{n}{2^{N-2}}\right) * g\left(\frac{n}{2^{N-1}}\right) * \ldots \\
& * g\left(\frac{n}{2^{i-j}}\right) * \ldots * g(n)
\end{aligned}
$$

where $(i-j)$ is a positive integer for $i, j \in 0,1, \ldots, N-2$.

The signal is up-sampled and filtered by the LPF coefficients or namely as approximated coefficients.

The system model in Figure 8 is assumed that there is no frequency offset so that the DWT itself acts as a matched filter at the receiver. To determine the data in sub-channel $k$, we match the transmitted waveform with carrier $i[17]$ :

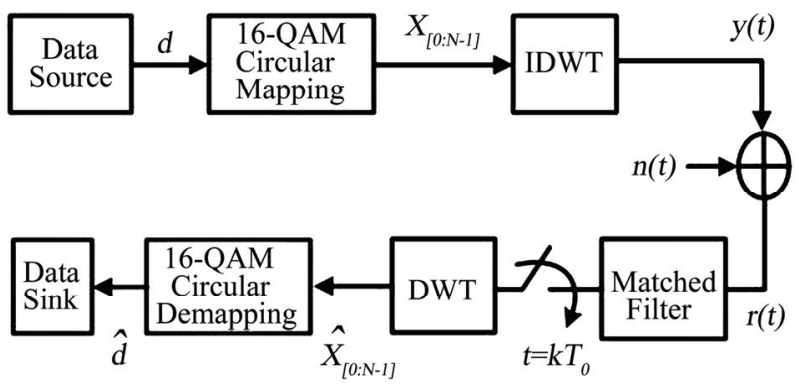

Figure 8. The system model of wavelet based OFDM transceiver. 


$$
\left\langle y(t), W_{i}()\right\rangle=\sum_{k=0}^{N-1} d_{k}\left\langle W_{k}(t), W_{i}(t)\right\rangle
$$

where $y(t)$ is the transmitted data via IDWT, $d_{k}$ is the data projected onto each carrier, $W_{k}(t)$ are the complex exponentials or it can be written as $e^{j 2 \pi 2 \frac{\mathrm{m}}{\mathrm{N}}}$, $\left\langle W_{k}(t), W_{i}(t)\right\rangle$ equals 1 when $k=i$ and 0 when $k \neq i$.

In a typical communication system, data is transmitted over a dispersive channel. The impulse response of a deterministic (and possibly time-varying) channel can be modeled by a linear filter $h(t)$ :

$$
\begin{aligned}
r(t) & =y(t) * h(t)+n(t) \\
& =\sum_{k=0}^{N-1} d_{k} W_{k}^{\prime}+\sum_{l=0}^{g-1} \sum_{k=0}^{N-1} d_{k, l} W_{k}^{\prime}(t-l k)+n(t)
\end{aligned}
$$

where $\mathrm{W}_{\mathrm{k}}^{\prime}=W_{k}(t) * h(t)$, and $g(g>1)$ is the wavelet genus so that $N g$ is the filter order (number of taps in that subband), and $n(t)$ is an additive white Gaussian noise. Due to the overlapped nature of the wavelet-based OFDM, it requires $g$ symbol periods, for a genus $g$ system, to decode one data vector [17]. This is the reason of having the wavelet transforms of $g-1$ other data vectors in the second term of (34).

After matched-filtering with carrier $i$, the signal becomes

$$
\begin{aligned}
\left\langle r(t), W_{i}(t)\right\rangle= & \sum_{k=0}^{N-1} d_{k}\left\langle W_{k}^{\prime}(t), W_{i}(t)\right\rangle \\
& +\sum_{l=1}^{g} \sum_{k=0}^{N-1} d_{k, l}\left\langle W_{k}^{\prime}(t-l k), W_{i}(t-l k)\right\rangle \\
& =\sum_{k=0}^{N-1} d_{k} \rho_{k, 0}(0)+\left\langle\left\langle n(t), f_{i}(t)\right\rangle\right\rangle \\
& =d_{k} \rho_{i, i}(0)+\sum_{k=0}^{N-1} d_{k} \rho_{k, i}(0) \\
& +\sum_{l=1}^{g} \sum_{\substack{k=0 \\
k \neq i}}^{N-1} d_{k, l} \rho_{k, i}(l)+n^{\prime \prime}(t)
\end{aligned}
$$

where $d_{k} \rho_{i, i}(0)$ is the recovered data with correlation term $\rho_{i, i}(0)$. The second term which is $\sum_{k=0, k \neq i}^{N-1} d_{k} \rho_{k, i}(0)$ is the interference due to the distorted filters that are no longer orthogonal to one another with correlation terms $\rho_{k, i}(0)$, and $\sum_{l=1}^{g} \sum_{k=0, k \neq i}^{N-1} d_{k, l} \rho_{k, i}(l)$ is the interference term with correlation $\rho_{k, i}(l)$ due to the overlapped nature of the wavelet transform. If the channel has no distortion, only the first and last terms would appear, which result that the decoder would obtain almost the correct signal.

\section{System Performance}

The performance between the square and circular for 16-QAM for unfiltered constellation points is discussed in Subsection 6.1. A filtered version, which is the processed signal through the matched filter and DWT block shown in Figure 8 at the receiver, is then considered. The result is obtained and discussed in Subsection 6.2.

\subsection{Square versus Circular}

In this subsection, two main parts are discussed. The first part is to obtain the simulation result for circular 16QAM from (16) and compare with the square 16-QAM provided by (17) in [4] which is written as follows

$$
\begin{aligned}
& P_{s q}= \frac{\sqrt{M}-1}{\sqrt{M} \log _{2}(\sqrt{M})} Q\left(\sqrt{\frac{3 \log _{2}(\sqrt{M}) \cdot E_{b}}{2(M-1) N_{0}}}\right) \\
&+\frac{\sqrt{M}-2}{\sqrt{M} \log _{2}(\sqrt{M})} Q\left(\sqrt{\frac{3 \log _{2}(\sqrt{M}) \cdot E_{b}}{2(M-1) N_{0}}}\right)
\end{aligned}
$$

Note that the square 16-QAM curve in Figure 9 is also approximately similar to the theoretical 16-QAM plot if one uses the Bit Error Rate Analysis Tool (bertool) from Matlab. From the figure, it is shown that the circular scheme slightly outperforms the counterpart scheme at most SNR values.

The second part is to obtain the result for other M-ary QAM. The exact BER analysis for other circular M-ary QAM is performed by changing the value of $M$ in $\mathrm{d}=\sqrt{\frac{3 \log _{2} \mathrm{M} \cdot \mathrm{E}_{\mathrm{b}}}{2(\mathrm{M}-1)}}$ and fix $\sigma$ accordingly. When $M$ is changed, the parameters $A, B$ and $C$ are consequently affected. Then, they are substituted into (16). Table I shows the summary of the arbitrary parameters due to varying $M$. Figure 9 also shows the BER results for other circular schemes with comparisons of other square QAM. The circular of other M-ary QAM are also slightly better than the square schemes in most SNR values. The simulation results show that they met the theoretical analysis.

\subsection{Wavelet Based OFDM}

To simulate the system using wavelet based OFDM (W-OF$\mathrm{DM})$, the orthogonal wavelet family such as Daubechies, 


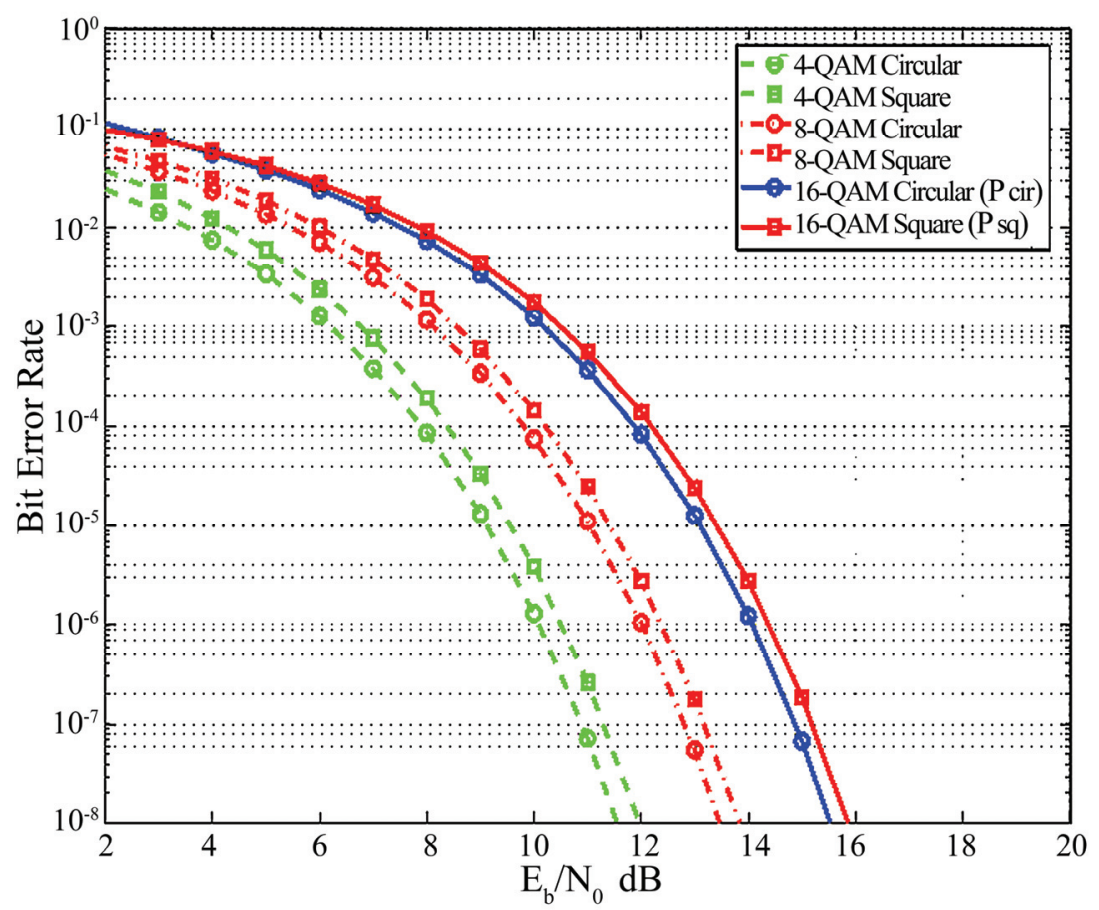

Figure 9. Exact BER of circular and square M-ary QAM.

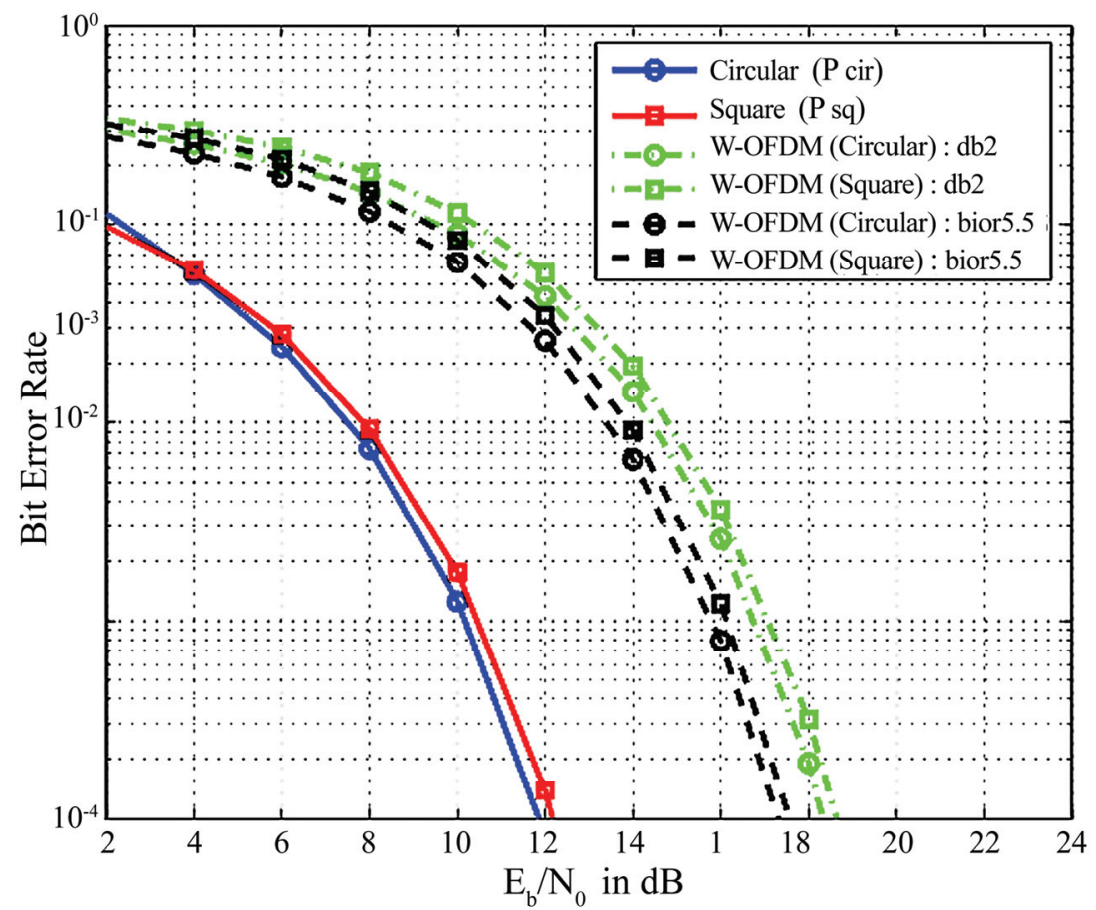

Figure 10. Comparison of circular and square of 16-QAM wavelet (db2 and bior5.5) and Fourier based OFDM.

$\mathrm{db} 2$ with comparison of the biorthogonal wavelet family, bior5.5 are considered. From Figure 10, it is shown that circular 16-QAM has better outperformed the square scheme in most SNR values. It is interesting to observe that the W-OFDMs results have less BER performance compared to $P_{c i r}$ and $P_{s q}$. The result is the effect of the filtered version that have been through the imperfect functional components in the receiver such as distorted filters and an additive white Gaussian noise channel as indicated in the previous section. 
Table 1. Summary of parameters for circular $M$-Ary $(M \leq$ 16) Qam.

\begin{tabular}{|c|c|c|c|}
\hline & $\mathrm{M}=4$ & $\mathrm{M}=8$ & $M=16$ \\
\hline$d$ & $\sqrt{E_{b}}$ & $\sqrt{\frac{9}{14} E_{b}}$ & $\sqrt{\frac{2}{5} E_{b}}$ \\
\hline$\gamma$ & $2 \sqrt{5 \frac{E_{b}}{N_{0}}}$ & $2 \sqrt{\frac{45}{14} \frac{E_{b}}{N_{0}}}$ & $2 \sqrt{2 \frac{E_{b}}{N_{0}}}$ \\
\hline$A$ & $Q\left(\sqrt{5 \frac{E_{b}}{N_{0}}}\right.$ & $Q\left(\sqrt{\frac{45}{14} \frac{E_{b}}{N_{0}}}\right.$ & $Q\left(\sqrt{2 \frac{E_{b}}{N_{0}}}\right.$ \\
\hline$B$ & $Q\left(b \sqrt{5 \frac{E_{b}}{N_{0}}}\right.$ & $Q\left(b \sqrt{\frac{45}{14} \frac{E_{b}}{N_{0}}}\right.$ & $Q\left(b \sqrt{2 \frac{E_{b}}{N_{0}}}\right.$ \\
\hline$C$ & $Q\left(c \sqrt{5 \frac{E_{b}}{N_{0}}}\right.$ & $Q\left(c \sqrt{\frac{45}{14} \frac{E_{b}}{N_{0}}}\right.$ & $Q\left(c \sqrt{2 \frac{E_{b}}{N_{0}}}\right.$ \\
\hline
\end{tabular}

Note: $b=0.464, c=2.464$ and $Q()=.\operatorname{erfc}($.

\section{Conclusions}

The optimal circular 16-QAM constellation points and the analysis of its exact BER calculation have been derived. The work has also been applied to wavelet based OFDM systems to compare the circular and square schemes. The results showed that the circular were slightly better than the square counterparts. When applying wavelet based OFDM using different wavelet families (orthogonal and biorthogonal), the same results were also obtained that the circular has slightly outperformed the square.

\section{References}

[1] J. G. Proakis, "Digital communications," Fourth edition, New York: McGraw-Hill, 2001.

[2] R. V. Nee and R. Prasad, "OFDM for wireless multimedia communications," Boston: Artech House, 2000.

[3] M. P. Fitz and J. P. Seymour, "On the bit error probability of QAM modulation," International Journal of Wireless Information Networks, Vol. 1, No. 2, pp. 131-139, 1994.
[4] K. Cho and D. Yoon, "On the general BER expression of one and two dimensional amplitude modulations," IEEE Transactions on Communications, Vol. 50, No. 7, pp. 1074-1080, July 2002.

[5] I. Daubechies, "Ten lectures on wavelets," Philapdelphia: Society for Industrial and Applied Mathematics, 1992.

[6] M. Weeks, "Digital signal processing using matlab and wavelets," Infinity Science Press LLC, 2007.

[7] C. S. Burrus, R. A. Gopinath, and H. Guo, "Introduction to wavelets and wavelet transforms," Upper Sadle River, NJ: Prentice-Hall, 1998.

[8] R. Mirghani and M. Ghavami, "Comparison between wavelet-based and Fourier-based multicarrier UWB systems," IET Communications, Vol. 2, No. 2, pp. 353-358, 2008.

[9] S. D. Sandberg and M. A. Tzannes, "Overlapped discrete multitone modulation for high speed copper wire communications," IEEE Journal on Selected Areas in Communications, Vol. 13, No. 9, pp. 1571-1585, 1995.

[10] F. Xiong, "Digital modulation techniques," Second edition, Boston: Artech House, 2006.

[11] I. Daubechies, "Orthonormal bases of compactly supported wavelets," Communications in Pure and Applied Math., Vol. 41, pp. 909-996, 1988.

[12] A. N. Akansu, "Wavelets and filter banks: A signal processing perspective," Tutorial in Circuit and Devices, November 1994.

[13] L. Cui, B. Zhai, and T. Zhang, "Existence and design of biorthogonal matrix-valued wavelets," Nonlinear Analysis: Real World Applications, Vol. 10, pp. 2679-2687, 2009.

[14] R. M. Rao and A. S. Bopardikar, "Wavelet transforms: Introduction to theory and applications," MA: AddisonWesley, 1998.

[15] R. K. Young, "Wavelet theory and its applictions," Massachusetts: Kluwer Academic, 1993.

[16] B. G. Negash and H. Nikookar, "Wavelet based OFDM for wireless channels," Vehicular Technology Conference, 2001.

[17] N. Ahmed, "Joint detection strategies for orthogonal frequency division multiplexing," Dissertation for Master of Science, Rice University, Houston, Texas, pp. 1-51, April 2000. 